\title{
An approximate solution to Prandtl's equations for a laminar flow over an infinite horizontal flat plate
}

\author{
J. Venetis \\ School of Applied Mathematics and Physical Sciences \\ Section of Mechanics \\ National Technical University of Athens, Greece \\ Email: johnvenetis4@gmail.com
}

\begin{abstract}
In this paper, a further mathematical analysis of some previous results of the author's ongoing investigation regarding Prandtl's equations is accomplished. In particular, the objective of the present work is the performance of an approximate explicit solution to Prandtl's system of equations for a laminar, isothermal, incompressible and steady boundary layer flow of a Newtonian fluid, over an infinite horizontal flat plate.
\end{abstract}

\section{Keywords}

boundary layer flow, flat plate, Prandtl's equations

\section{Introduction}

The first exact analytical solution to Prandtl's equations for an incompressible, steady and laminar flows over a horizontal planar surface was obtained from Blasius by means of a combination of differential and dimensional analysis of the flow field [1]. The main assumption of this method is that the dimensionless parameter $V_{x} / V_{\infty}$ depends on the horizontal transposition. Here, let us remark that $V_{\infty}$ denotes free stream velocity. Also, the same fundamental assumption was adopted by Falkner Skan approach of similar boundary layer flows [2,3]. In the meanwhile, in opposition with Blasius solution which is exact, Karman and Pohlhausen method towards an approximate solution to Prandtl's Equations, assumes an approximate shape of the velocity profile [4]. This method, which is also known as Momentum Integral Method, changes the two Prandtl's partial differential equations into a single differential equation which is applicable only when the pressure gradient term is zero. Besides, a remarkable progress was made, concerning the existence and the properties of analytical solutions to unsteady Prandtl's equations [5,6,7]. On the other hand, an alternative exact closed - from solution to these equations was proposed by Venetis et al. without dimensional arguments and functional notations with respect to the main characteristic sizes of the flow, (e.g. Re number) [8]. Further, in Ref. [9] the obtaining of an approximate explicit solution to Prandtl's equations for isothermal, incompressible and steady boundary layer flows of Newtonian fluids, in a coordinate free context was performed, whilst for a detailed study on the stability of Prandtl's boundary layers one may refer to a valuable work by Grenier et al. [10]. In Ref. [11] rigorous closed form solutions to unsteady free convection flow of a second srade fluid over an oscillating vertical plate were derived, whereas a thorough investigation on unsteady convection flows over a moving vertical flat plate was carried out in Ref. [12]. A similar remarkable study on unsteady natural convection flows past a vertical infinite flat plate was performed in Ref. [13], while in Ref. [14] an important computational treatment to Prandtl-Blasius laminar viscous flow over a semi-infinite flat plate can be found.

In Ref. [15], a theoretical investigation of a steady boundary layer flow near the inflection point of a smooth mathematical curve was exhibited, whereas a prominent study on magneto-hydro-dynamic (MHD) convection flows over a stretched vertical flat plate was performed in Ref. [16]. In Ref. [17] a useful computational approach to 2D laminar forced convective viscous flows over a flat plate was carried out, whilst an analytical solution of forced-convective boundary-layer flow over a flat plate was proposed in Ref. [18]. In Ref. [19] an implicit finite difference solution of a boundary layer heat 
flow past a flat plate was derived, whilst for a detailed study on the role of pressure gradient on laminar boundary layer over a permeable surface with convective boundary conditions, one may refer to Ref. [20].

The intention of the present work is the obtaining of an approximate explicit solution to Prandtl's equations for laminar, isothermal, incompressible and steady boundary layer flows of Newtonian fluids over a horizontal infinite flat plate. To this end, closed - form solutions to these equations were derived on the basis of Ref. [9]. Here, we have to emphasize that some simplifications were primarily taken into account in order to obtain these explicit expressions. These simplifications arise from Prandtl's simplified assumptions for boundary layer flows.

\section{Towards an approximate solution to Prandtl's equations}

Let us write out Prandtl's equations in a Cartesian frame of reference positioned at an arbitrary point of an infinite horizontal flat plate.

$$
\begin{aligned}
& \frac{\partial V_{x}}{\partial x}+\frac{\partial V_{y}}{\partial y}=0 \\
& V_{x} \frac{\partial V_{x}}{\partial x}+V_{y} \frac{\partial V_{x}}{\partial y}=-\frac{1}{\rho} \cdot \frac{d P}{d x}+\frac{\mu}{\rho} \cdot \frac{\partial^{2} V_{x}}{\partial y^{2}}
\end{aligned}
$$

Evidently the term $P$ which is supposed to depend only on the variable $x$, denotes the algebraic sum of barometric and manometric pressure. In addition, for isothermal incompressible boundary layer flows of a Newtonian fluid, the known from the literature Prandtl's assumptions hold [1]:

$$
\begin{aligned}
& V_{y} \ll<V_{x} \\
& \frac{\partial V_{x}}{\partial x}<<\frac{\partial V_{x}}{\partial y} \\
& \frac{\partial^{2} V_{x}}{\partial x^{2}}<<\frac{\partial^{2} V_{x}}{\partial y^{2}} \\
& P=P(x)
\end{aligned}
$$

Here, the pressure $P(x)$ is estimated by the following relationship

$$
\frac{d P(x)}{d x}=\rho \cdot V_{\infty} \cdot \frac{d V_{\infty}}{d x}
$$

where $V_{\infty}$ denotes the free stream velocity, which is assumed to arise from a potential flow field [1]. In this context, eqn. (7) informs us that the gradient of pressure is defined by means of a frictionless flow field. This implies that all the sequential velocity distributions are similar in shape to one another at different axial locations, having changes only as concerns the quantity scale across boundary layer thickness. In this framework, the geometry of the flow field inside boundary layer can be formulated equivalently without violating the nature of the problem, as a sequence of transverse cross - sections of the horizontal or inclined surface, which correspond injectively to sequential rates of the longitudinal displacement measured from the point where the rectangular Cartesian frame of reference has been positioned. In Ref. [9], it was shown that the governing velocity component along such a boundary layer can be approximated as

$$
V_{x}(x, y)=\frac{\mathrm{a} \cdot y^{2}}{3}+2 h(x-y)+h(x+2 y)-\mathrm{a} \int_{x-y}^{x+2 y} f(\lambda) d \lambda+g(x)
$$

Here, $f, h, g$ are arbitrary continuous single valued functions and a is an arbitrary constant.

Now, let us remark that since $V_{y}(x, y)$ is not the governing velocity component, its variations do not affect the flow in a significant manner. In Ref. [9], for facility reasons, it was assumed that $V_{y}(x, y)$ obeys a linear variation law, i.e.

$V_{y}(x, y)=C_{1} x+C_{2} y+C_{3}$ 
In addition, it is known [1] that the flow field inside boundary layer is formulated as a sequence of transverse cross - sections of the planar surface and therefore the independent variable $x$ can be generally considered as a parameter. Concurrently, according to Prandtl's assumptions it is valid that $V_{y}<<V_{x}$.

Nonetheless, one may point out that a shortcoming of eqn. (8) is the existence of three arbitrary single valued functions i.e. $h, f, g$.

In the present investigation, in order to overcome this issue let us suppose that the following relationship holds

$\left(\frac{f}{h}\right)(t) \equiv \frac{f(t)}{h(t)}=Q(t), \forall t \in R$

where $Q$ is an nth degree polynomial

By making the additional assumption that the single valued continuous functions $f, h$ are analytic over their domain of definition and thus are able to be expanded in Taylor series, one may deduce that the validity of eqn. (10) does not violate the generality.

In continuing, to approach the single valued function $h(t)$ let us consider that it may have a general exponential and a trigonometric form respectively. Hence we can write out

$h(t)=b \cdot e^{A t}$

or

$h(t)=b \cdot \cos (A t)$

with $A, b$ are arbitrary constants

On the basis on eqns. (10) and (11), the calculation of the indefinite integral of the form $\int e^{A t} Q(t) d t$ is necessary to obtain the modified form of eqn. (8).

This integral can be estimated as

$\int e^{A t} Q(t) d t=\frac{e^{A t}}{A} \cdot\left(Q(t)-\frac{1}{A} \cdot Q^{(1)}(t)+\frac{1}{A^{2}} \cdot Q^{(2)}(t)-\frac{1}{A^{3}} \cdot Q^{(3)}(t)+\frac{1}{A^{4}} \cdot Q^{(4)}(t)-\ldots+(-1)^{n} \frac{1}{A^{n}} \cdot Q^{(n)}(t)\right)+C(13)$

Here we took into account that the following relation holds identically

$Q^{(n+1)}(t) \equiv 0$

Eqn. (13) can be represented in a more elegant manner as follows

$$
\int e^{A t} Q(t) d t=\frac{e^{A t}}{A} \cdot \sum_{0}^{n}(-1)^{n} \frac{1}{A^{n}} \cdot Q^{(n)}(t)+C
$$

Thus, eqn. (8) can be combined with eqn. (11) and (15) to yield

$$
V_{x}(x, y)=\frac{\mathrm{a} \cdot y^{2}}{3}+\frac{2 e^{-y}+e^{2 y}}{e^{-A x}}+\frac{\mathrm{a} \cdot b \cdot e^{A x}}{A}\left(e^{-y} \sum_{0}^{n}(-1)^{n} \frac{1}{A^{n}} \cdot Q^{(n)}(x-y)-e^{2 y} \sum_{0}^{n}(-1)^{n} \frac{1}{A^{n}} \cdot Q^{(n)}(x+2 y)\right)+g(x)(16)
$$


Next, by requesting $V_{x}(x, 0)=0$ it follows

$$
\begin{aligned}
& \sum_{0}^{n}(-1)^{n} \frac{1}{A^{n}} \cdot Q^{(n)}(x)-\sum_{0}^{n}(-1)^{n} \frac{1}{A^{n}} \cdot Q^{(n)}(x)=\left(\frac{g(x)}{e^{A x}}+3\right) \frac{A}{\mathrm{a} \cdot \mathrm{b}} \Rightarrow \\
& g(x)=-3 e^{A x}
\end{aligned}
$$

Here one may observe that the continuous function $g$ is independent of the coefficients and the degree of the polynomial $Q$ but indeed depends on the selection of the continuous function $h$.

Then, eqn. (16) can be combined with eqn. (17) to yield

$$
V_{x}(x, y)=\frac{\mathrm{a} \cdot y^{2}}{3}+\frac{2 e^{-y}+e^{2 y}}{e^{-A x}}+\frac{\mathrm{a} b \cdot e^{A x}}{A}\left(e^{-y} \sum_{0}^{n}(-1)^{n} \frac{1}{A^{n}} \cdot Q^{(n)}(x-y)-e^{2 y} \sum_{0}^{n}(-1)^{n} \frac{1}{A^{n}} \cdot Q^{(n)}(x+2 y)\right)-3 e^{A x}
$$

On the other hand, to obtain the final form of eqn. (8) on the basis of eqns. (10) and (11), the analytical evaluation of the indefinite integral of the form $\int \cos (A t) Q(t) d x, \forall A \in R^{*}$ is necessary. This integral can be estimated as

$$
\int \cos (A t) Q(t) d t=\frac{1}{A} \cdot\left(\begin{array}{l}
\sin (A \cdot t) Q(t)+\frac{1}{A} \cdot Q^{(1)}(t) \cdot \sin \left(A \cdot t+1 \cdot \frac{\pi}{2}\right)+\frac{1}{A^{2}} \cdot Q^{(2)}(t) \cdot \sin \left(A \cdot t+2 \cdot \frac{\pi}{2}\right)+\ldots \\
+\frac{1}{A^{n}} \cdot Q^{(n)}(t) \sin \left(A \cdot t+n \cdot \frac{\pi}{2}\right)
\end{array}\right)+C_{1}
$$

Again, we took into account that $Q^{(n+1)}(t) \equiv 0$.

Eqn. (19) can be represented in a more elegant manner as follows

$$
\int \cos (A t) Q(t) d t=\frac{1}{A} \cdot \sum_{0}^{n}\left(\frac{1}{A^{n}} \cdot Q^{(n)}(t) \sin \left(A \cdot t+n \cdot \frac{\pi}{2}\right)\right)+C_{1}
$$

Next, eqn. (8) can be combined with eqn. (12) and (20) to yield

$$
\begin{aligned}
V_{x}(x, y)= & \frac{\mathrm{a} y^{2}}{3}+2 b \cos (A(x-y))+b \cos (A(x+2 y))-\frac{\mathrm{ab}}{A} \sum_{0}^{n}\left(\frac{1}{A^{n}} \cdot Q^{(n)}(x+2 y) \sin \left(A \cdot(x+2 y)+n \cdot \frac{\pi}{2}\right)\right)+ \\
& +\frac{\mathrm{ab}}{A} \sum_{0}^{n}\left(\frac{1}{A^{n}} \cdot Q^{(n)}(x-y) \sin \left(A \cdot(x-y)+n \cdot \frac{\pi}{2}\right)\right)+g(x)
\end{aligned}
$$

In addition, by requesting $V_{x}(x, 0)=0$ it implies that

$g(x)=-3 b \cos (A x)$

Again, one may pinpoint that the continuous function $g$ is independent of the coefficients and the degree of the polynomial $Q$ but indeed depends on the selection of the function $h$.

Then, eqn. (21) can be combined with eqn. (22) to yield

$$
\begin{aligned}
V_{x}(x, y)= & \frac{\mathrm{a} y^{2}}{3}+2 b \cos (A(x-y))+b \cos (A(x+2 y))-\frac{\mathrm{ab}}{A} \sum_{0}^{n}\left(\frac{1}{A^{n}} \cdot Q^{(n)}(x+2 y) \sin \left(A \cdot(x+2 y)+n \cdot \frac{\pi}{2}\right)\right)+ \\
& +\frac{\mathrm{ab}}{A} \sum_{0}^{n}\left(\frac{1}{A^{n}} \cdot Q^{(n)}(x-y) \sin \left(A \cdot(x-y)+n \cdot \frac{\pi}{2}\right)\right)-3 b \cos (A x)
\end{aligned}
$$




\section{Discussion}

The objective of this paper was the obtaining of approximate explicit solutions to Prandtl's equations for laminar isothermal, incompressible and steady boundary layer flows of Newtonian fluids over a horizontal infinite flat plate. To this end, closed - form solutions to these significant equations were derived on the basis of the mathematical analysis appeared in Ref. [9]. However, we have to emphasize that some simplifications were primarily taken into account in order to obtain these analytical representations. These fundamental simplifications are motivated by Prandtl's assumptions for boundary layer flows. Therefore, to implement this closed - form expression to a boundary layer flow one must always be ensured whether these expressions hold. Also, we have to elucidate that the condition of uniform flow at infinity cannot be fulfilled by means of the proposed explicit expressions, i.e. eqns. (18) and (23). In this context, one has definitely to verify experimentally this concept before taking into consideration these relationships. Moreover, it should be clarified that the well known non - slip condition, which was taken into account, cannot be explained and justified only by the existence of the cohesion. Therefore, it is necessary to invoke the molecular, i.e. the discontinuous structure of the matter for the solid bounds as well as for the fluid. Indeed, the macroscopically appearing as smooth surface of a solid rigid body has cavitational irregularities in size order larger than that of the molecule. In this context, the molecules of the fluid which during their uncertain molecular motion strike against this surface loosing their macroscopic velocity, i.e. flow velocity. Now, according to the moleculokinetic interpretation of the cohesion, the molecules which escape from the irregularities of the solid wall and reenter in the flow, strike against the molecules that pass with the flow velocity and they accelerate with simultaneous deceleration of those which move beyond the wall. Hence, the velocity from zero on the solid wall increases away from it until settles the value that would be imposed by the solution of the corresponding potential flow.

\section{Conclusions}

In this article, a further mathematical analysis of some previous results of the author's ongoing investigation regarding Prandtl's equations was performed. Specifically, the aim of this work aimed at presenting in a rigorous manner an approximate explicit solution to Prandtl's system of equations for laminar isothermal, incompressible and steady boundary layer flows of Newtonian fluids over a horizontal and infinite flat plate. Nonetheless, we have to elucidate that a shortcoming of the proposed explicit formulae is that the condition of uniform flow at infinity cannot be fulfilled.

\section{References}

1. H. Schlichting, Boundary Layer Theory (7th Edition)McGraw-Hall, New York (1987)

2. V.M. Falkner, B. Scan, Solutions of the boundary-layer equation, Philosophical Magazine Series 7, Volume 12, No. 80, pages, 865-896, 1931

3. L. Howarth, On the Solution of the Laminar Boundary Layer Equations, Proceedings of the Royal Society of London. Series A, Mathematical and Physical Sciences, Vol. 164, No. 919, 1938, pp. 547579

4. T. Von Karman, C. Millikan, On the theory of laminar boundary layr separation, N.A.C.A. Report No. 504, 1934.

5. O. Oleinik, On the mathematical theory of boundary layer for unsteady flow of incompressible fluid, Journal of Appl. Math. Mech. Vol. 30, 1966, pp. 951-974.

6. E. Weinan,B., B. Enquist, Blow up of solutions of the unsteady Prandtl's equation Comm. Pure Appl. Math., Vol. 50, 1998, pp. 1287-1293 
7. Zhouping Xin, Liqun Zhang, On the global existence of solutions to the Prandtl's system, Advances in Mathematics, Vol. 181, No. 1, 2004, pp. 88-133

8. J. Venetis, E.Sideridis, On a Closed - Form Solution of Prandtl's System of Equations, International journal of fluid mechanics research Vol. 40, No. 2, 2013, pp. 106-114

9. J. Venetis, An approximate explicit solution to Prandtl's system of equations, Far East Journal of Mathematical Sciences, Vol. 84, No. 1, 2014, pp. 1-11

10. Emmanuel Grenier, Yan Guo, Toan T. Nguyen Spectral stability of Prandtl boundary layers: An overview, Analysis, Volume 35, Issue 4, Pages 343-355, 2015

11. Ali F, Khan I, Shafie S Closed Form Solutions for Unsteady Free Convection Flow of a Second Grade Fluid over an Oscillating Vertical Plate, PLoS ONE Vol. 9 No. 2 2014, Article ID: e85099.

12. M. Turkyilmazoglu, Unsteady Convection Flow of Some Nanofluids Past a Moving Vertical Flat Plate With Heat Transfer J. Heat Transfer., 2014, Vol. 136, No. 3 : Article ID 031704 (7 pages)

13. M. Turkyilmazoglu I.Pop, Heat and mass transfer of unsteady natural convection flow of some nanofluids past a vertical infinite flat plate with radiation effect, International Journal of Heat and Mass Transfer, Volume 59, 2013, Pages 167-171

14. K. Parand, M. Nikarya, J. Rad, F. Baharifard, A new Reliable Numerical Algorithm Based on the First Kind of Bessel Functions to Solve Prandtl-Blasius Laminar Viscous Flow over a Semi-Infinite Flat Plate. Zeitschrift für Naturforschung A, Vol. 67, No. 12, 2014, pp. 665-673

15. J. Venetis, Investigation of a Boundary Layer Flow near the Inflection Point of a Smooth Curve Advances in Energy and Power Vol. 2, No. 2, 2014, pp. 13-20

16. Gnaneswar Reddy Machireddy, Influence of thermal radiation, viscous dissipation and Hall current on MHD convection flow over a stretched vertical flat plate, Ain Shams Engineering Journal, Volume 5, No. 1, 2014, pp. 169-175

17. Jaionto Karmokar, Md. Sayeed, Iftekhar Yousuf, M. M. Billah, and M. A. Haque, The Numerical Analysis of 2D Laminar Forced Convective Flow of Viscous Fluid over a Flat Plate, Journal of Computer and Mathematical Sciences, Vol. 10, No. 3, 2019, pp. 524-533

18. H. Mirgolbabai, A. Barari, and G. Domiri, Analytical solition of forced-convective boundary-layer flow over a flat plate, Archive of Civil and Mechanical Engineering Vol. 10, No. 2, 2010, pp. 42-51.

19. S. V. Desale, V. H. Pradhan, Implicit Finite Difference Solution of Boundary Layer Heat Flow over a Flat Plate. Int. J. Eng. Res. Appl. Vol. 3, No. 6, 2013, pp. 1611-1616.

20. Y. S. Daniel, S. K. Daniel, Presence of Pressure Gradient on Laminar Boundary Layer over a Permeable Surface with Convective Boundary Condition, American Journal of Heat and Mass Transfer, Vol. 2, No. 1, 2015, pp. 1-14 